\title{
Avaliação do impacto de veículos pesados na qualidade de serviço de rodovias de pista dupla usando dados empíricos
}

\author{
Fernando José Piva ${ }^{1}$, José Reynaldo Setti ${ }^{2}$
}

\begin{abstract}
Resumo: Este trabalho visa avaliar o impacto de veículos pesados na qualidade de serviço de correntes de tráfego a partir de estimativas dos fatores de equivalência veicular em rodovias com três ou mais faixas de tráfego por sentido, obtidas a partir de dados empíricos coletados em rodovias paulistas, para intervalos de duração curta (5 ou 6 minutos). O fator de equivalência foi calculado para cada intervalo através do método de Huber, admitindo-se que a qualidade de serviço é a mesma para todas as faixas de tráfego naquele intervalo. O fluxo básico é o da faixa mais à esquerda, nos intervalos em que são detectados apenas automóveis, e o fluxo misto é o da faixa mais à direita, em que passam automóveis e caminhões. Os resultados obtidos sugerem que: (1) em 52\% do tempo, a qualidade de serviço não é a mesma em todas as faixas da rodovia; (2) o impacto marginal dos caminhões decresce à medida em que a porcentagem de caminhões na corrente aumenta; e (3) as diferenças que existem no fator de equivalência em função do nível de serviço são menos evidentes em rampas mais íngremes, onde o efeito das limitações de desempenho dos caminhões é mais notado.
\end{abstract}

Palavras-chave: rodovias de pista dupla, qualidade de serviço, fator de equivalência, dados empíricos.

\begin{abstract}
In this paper, the impact of heavy vehicles on the quality of service is studied by means of PCE estimates obtained from empirical data collected on divided highways in the state of São Paulo, for short time intervals (5 or 6 minutes). PCE estimates were calculated for each time interval using Huber's method, assuming that the quality of service is the same for all traffic lanes during a given interval. The leftmost lane represented the basic flow (cars only) for the intervals in which no trucks are detected on the left lane, whereas observed flow on the rightmost lane was the mixed flow (trucks and cars). The results suggest that: (1) in 52\% of the time the quality of service is not the same in all traffic lanes; (2) the marginal impact of trucks decreases as the number of trucks in the traffic stream increases; and (3) differences in PCE due to the level of service become less noticeable in steeper up grades, where truck loss of speed due to poor performance characteristics is greater.
\end{abstract}

Keywords: Expressways, quality of service, passenger-car equivalents, traffic data.

\section{INTRODUÇÃO}

Para verificar os níveis de serviço dos diferentes trechos de rodovia e assim identificar onde devem ser efetuadas eventuais aumento de faixas pelos concessionários privados, os órgãos reguladores das rodovias brasileiras utilizam parâmetros de diferentes versões do HCM, como 2000, 1994 e até 1985 [Riente et al., 2011]. Porém, a utilização direta, sem adaptações, do HCM no Brasil pode não apresentar resultados confiáveis [Setti, 2009].

O fator de equivalência veicular é utilizado para estimar o número de automóveis que são representados por um veículo pesado em uma corrente de tráfego. Através da sua multiplicação pelo número de veículos pesados existentes em uma contagem veicular, é possível obter uma corrente de tráfego hipotética, em que existem apenas veículos de passeio. Essa corrente é utilizada para analisar o nível de serviço e a capacidade da rodovia pelo método do HCM (Highway Capacity Manual) [TRB, 2010].

Este trabalho visa avaliar o impacto de veículos pesados em correntes de tráfego, a partir de estimativas dos fatores de equivalência veicular em rodovias com três ou mais faixas de tráfego. Essas estimativas foram obtidas a

\footnotetext{
${ }^{1}$ Fernando José Piva, Departamento de Engenharia de Transportes, USP. (fjpiva@usp.br)

2 José Reynaldo Setti, Departamento de Engenharia de Transportes, USP (jrasetti@usp.br)

Manuscrito recebido em 16/06/2015 e aprovado para publicação em $17 / 08 / 2015$

Este artigo é parte de TRANSPORTES v. 23, n. 4, 2015. ISSN: 2237-1346

(online). DOI: 10.14295/transportes.v23i4.949
}

partir de dados empíricos coletados em 8 estações permanentes de monitoramento localizadas em rodovias do estado de São Paulo.

\section{REVISÃO DA LITERATURA}

O fator de equivalência veicular é usado em diversas áreas da engenharia de transportes, para transformar uma população formada por vários tipos de veículos numa população uniforme, composta por um veículo padrão, visando facilitar a análise de um problema. A forma de calcular o fator de equivalência depende do propósito de cada tipo de análise.

$\mathrm{Na}$ análise da operação de rodovias e vias urbanas, o veículo padrão é o carro de passeio. Caminhões, ônibus e motocicletas são convertidos em carros de passeio equivalentes (cpe) através de fatores de equivalência. A estimativa de valores para esses fatores de equivalência pode ser feita com base no headway, no atraso, na formação de pelotões, na velocidade, no tipo de veículo, no fluxo (veic/hora) ou no tempo de viagem [Elefteriadou et al., 1997].

Em estudos de capacidade e nível de serviço, os volumes de tráfego observados nas rodovias devem ser convertidos para a unidade padrão, cpe/(h.faixa), utilizando-se a expressão

$$
v_{p}=\frac{V}{P H F \cdot N \cdot f_{H V} \cdot f_{p}}
$$

em que $v_{p}$ é a taxa de fluxo equivalente, em cpe/(h.faixa); $V$ é o volume horário observado, em veic/h; 


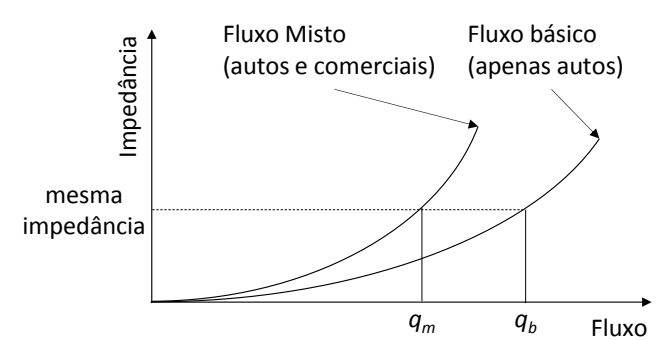

Figura 1. Conceito de equivalência entre uma corrente de tráfego básica e mista [Huber, 1982]

$P H F$ : é o fator de hora pico; $N$, o número de faixas de tráfego; $f_{H V}$, o fator de ajuste para veículos pesados; e $f_{p}$ é o fator de ajuste para o tipo de motorista.

Para realizar o cálculo na Equação (1), é necessário calcular o fator de ajuste para veículos pesados. Utilizandose o fator de equivalência veicular, é possível converter caminhões, ônibus e veículos de recreação em carros de passeio, através da expressão

$$
f_{H V}=\frac{1}{1+P_{T}\left(E_{T}-1\right)+P_{R}\left(E_{R}-1\right)}
$$

em que $P_{T}$ é a fração de caminhões e ônibus na corrente de tráfego; $P_{R}$ é a fração de veículos de recreação na corrente de tráfego; $E_{T}$, o fator de equivalência de um caminhão ou ônibus na corrente de tráfego; e $E_{R}$ é o fator de equivalência de um veículo de recreação na corrente de tráfego.

O HCM [TRB, 2010] apresenta tabelas com o fator de equivalência veicular para caminhões, ônibus e veículos de recreação para trechos com diferentes declividades, comprimento dos aclives e porcentagens de veículos pesados na corrente de tráfego. Dessa forma, é possível converter o fluxo misto, composto por automóveis e veículos comerciais, em fluxo básico (composto por automóveis) e utilizar as curvas velocidade-fluxo para os diferentes segmentos de rodovias.

Diversos estudos adotam uma abordagem em que o cálculo de fatores de equivalência veicular é feito através da comparação de duas correntes de tráfego com a mesma qualidade de serviço, uma formada por automóveis e caminhões (chamada de corrente mista) e a outra, contendo apenas automóveis, chamada de corrente básica. A determinação da qualidade de serviço na corrente de tráfego depende da abordagem escolhida em cada estudo. Para Linzer et al. [1979], duas correntes de tráfego com a mesma relação $v / c$ apresentam a mesma qualidade de serviço. Huber [1982] estima os fatores pela razão entre fluxos de duas correntes em um mesmo nível de impedância, que pode ser medida por indicadores de desempenho como velocidade e densidade. Sumner et al. [1984] generalizaram o método de Huber para incluir explicitamente mais de um tipo de caminhão na corrente de tráfego, de forma a ser possível calcular o fator de equivalência para cada tipo de caminhão. A ordem em que cada classe de caminhões é incorporada à corrente mista, entretanto, afeta o valor do seu equivalente veicular, conforme demonstrado por Demarchi e Setti [2003].

\subsection{Método de Huber}

Os fatores de equivalência podem ser estimados de acordo com o método proposto por Huber [1982], no qual o cálculo dos veículos equivalentes pode ser feito através da relação entre os fluxos de duas correntes de tráfego, uma contendo caminhões trafegando junto com automóveis (fluxo misto) e outra corrente de tráfego apenas com automóveis (fluxo básico), considerando-se que as duas correntes apresentam um mesmo nível de impedância. A Figura 1 ilustra a relação fluxo-impedância, com a representação das curvas do fluxo misto e básico.

A impedância deve estar relacionada com a qualidade de serviço na rodovia. Para um dado valor da impedância (ou seja, da qualidade de serviço), conhece-se o fluxo básico $q_{b}$ e o fluxo misto $q_{m}$. Admitindo-se que, para esses fluxos, as duas correntes de tráfego operam com qualidade de serviço idênticas, pode-se escrever que

$$
q_{b}=(1-p) q_{m}+p \cdot q_{m} \cdot E
$$

em que $q_{b}$ : fluxo básico, somente com automóveis; $p$ : fração de caminhões na corrente de tráfego; $q_{m}$ : fluxo misto, composto por $p$ caminhões e (1-p) automóveis; e $E$ : fator de equivalência dos caminhões na corrente de tráfego.

O método pode ser estendido para mais de uma classe de caminhões, ainda que variações na composição do tráfego de veículos pesados introduzam diferenças nos valores do equivalente veicular que podem não ser desprezíveis [Demarchi e Setti, 2003].

Tradicionalmente, valores para o fator de equivalência são obtidos através de simulação. O processo consiste em simular uma corrente de tráfego composta apenas por automóveis (fluxo básico) e uma outra corrente formada por automóveis e veículos pesados (fluxo misto). O uso de simulação facilita o processo, uma vez que é difícil observar, na vida real, fluxos compostos apenas por automóveis. Como se descreve a seguir, esta pesquisa usa o método proposto por Huber [1982] para estimar valores do fator de equivalência a partir de dados empíricos, obtidos de sensores instalados em rodovias de pista dupla.

\section{MÉTODO PROPOSTO}

A Figura 2 apresenta observações da velocidade média dos automóveis em função da taxa de fluxo de tráfego para intervalos de 6 minutos de duração obtidas no km 32N da SP348 (Rodovia dos Bandeirantes), estratificadas em função da faixa de tráfego (faixa 1 sendo a mais próxima do canteiro central e faixa 4, a faixa ao lado do acostamento). Comparando-se a composição de tráfego entre as quatro faixas de rolamento, verifica-se que a faixa 1 apresenta, em média, 99\% de automóveis, enquanto a faixa mais próxima ao acostamento (faixa 4) apresenta $85 \%$ de veículos pesados, na média.

Admitindo-se que a corrente de tráfego da faixa 1 (próxima ao canteiro central, representada por pontos em azul) representa a curva fluxo-velocidade de uma corrente veicular composta apenas por carros de passeio que seria observada neste trecho de rodovia se todas as faixas tivessem essa mesma composição de tráfego, as observações das outras faixas mostram claramente a impedância causada pela introdução de veículos pesados na corrente de tráfego. Admitindo-se que a qualidade de serviço é a mesma nas quatro faixas de tráfego no intervalo de tempo em que os dados de fluxo e velocidade foram coletados, é possível es- 
timar o valor do fator de equivalência para os veículos pesados a partir da velocidade e do fluxo observados em cada faixa.

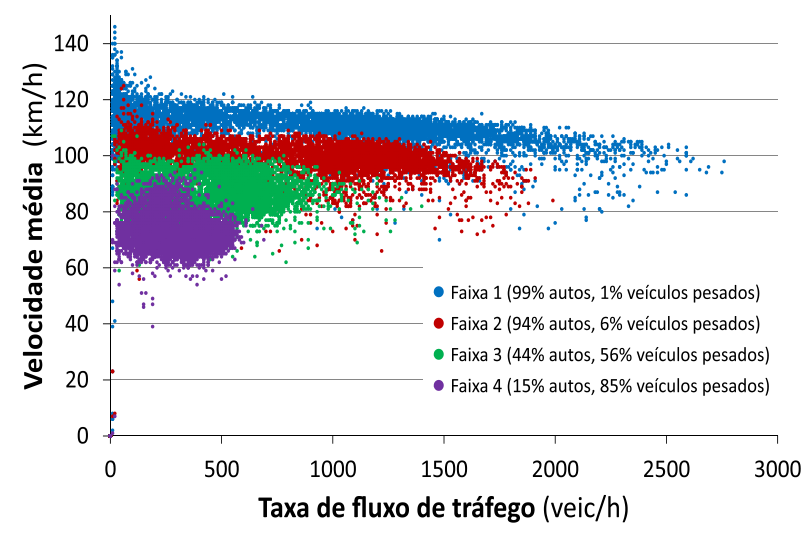

Figura 2. Velocidade média dos automóveis em função do fluxo, em cada faixa de tráfego, na SP348, km 32, Norte.

A abordagem adotada para estimar o fator de equivalência $E$ a partir de dados empíricos (como os mostrados na Figura 2) aplica o método proposto por Huber [1982]. Admita-se que, em um intervalo de tempo $i$, com duração curta (5 ou 6 minutos), observa-se um fluxo básico (composto apenas por automóveis) $q_{e}(i)$ na faixa da esquerda - a mais próxima do canteiro central. Admita-se também que, no mesmo intervalo de tempo $i$, observa-se um fluxo misto (composto por automóveis e veículos pesados) $q_{d}(i)$ na faixa da direita - por exemplo, na faixa ao lado do acostamento. Partindo-se da hipótese de que, durante esse intervalo $i$, a qualidade de serviço é a mesma em todas as faixas de rolamento, pode-se considerar que os dois fluxos $q_{e}(i)$ e $q_{d}(i)$ apresentam a mesma impedância, conforme pode ser visto na Figura 1. Definindo-se essa impedância como sendo a densidade, a hipótese proposta pode ser expressa pela igualdade

$$
k_{b d}(i)=k_{e}(i)
$$

em que $k_{b d}(i)$ é a densidade, em veículos equivalentes, da corrente de tráfego da faixa da direita (fluxo misto), durante o $i$-ésimo intervalo de tempo em cpe/(km.faixa); $k_{e}(i)$ é a densidade, em veículos equivalentes, da corrente de tráfego da faixa da esquerda (fluxo básico), durante o intervalo $i$, em cpe/(km.faixa); e $i$ é um intervalo de tempo de duração curta (5 ou 6 minutos).

Utilizando-se a equação fundamental do tráfego $(q=k \cdot u)$, pode-se reescrever a Equação (4):

$$
\frac{q_{b d}(i)}{u_{d}(i)}=k_{e}(i)
$$

em que $q_{b d}(i)$ é o fluxo, em veículos equivalentes, da corrente de tráfego da faixa da direita (fluxo misto), durante o intervalo $i$, em cpe/(h.faixa); $u_{d}(i)$ é a velocidade média da corrente de tráfego da faixa da direita, para o intervalo $i$, $\mathrm{em} \mathrm{km} / \mathrm{h}$.

O método de Huber [1982] pressupõe que a relação de equivalência entre o fluxo básico e o fluxo misto pode ser representada através da equação

$$
q_{b d}(i)=q_{d}(i) \cdot[1-p(i)]+E(i) \cdot p(i) \cdot q_{d}(i)
$$

em que $q_{d}(i)$ é a taxa de fluxo da corrente de tráfego da faixa da direita (fluxo misto), no intervalo $i$, em TRANSPORTES v. 23, n. 4 (2015), p. 51-59 veic/(h.faixa); $E(i)$ é o fator de equivalência veicular para o intervalo $i ; p(i)$ é a fração de veículos pesados na faixa da direita (fluxo misto) no intervalo $i$; e $[1-p(i)]$ é a fração de automóveis no fluxo composto, no intervalo $i$.

Substituindo-se a Equação (6) em (5), pode-se deduzir que:

$$
\begin{aligned}
& k_{e}(i)=\frac{q_{d}(i)[1-p(i)]+E(i) \cdot p(i) \cdot q_{d}(i)}{u_{d}(i)} \Rightarrow \\
& k_{e}(i)=\frac{q_{d}(i)}{u_{d}(i)}[E(i) \cdot p(i)+1-p(i)] .
\end{aligned}
$$

Pela equação fundamental do tráfego $(k=q / u)$, obtém-se a equação:

$$
k_{d}(i) \cdot[E(i) \cdot p(i)+1-p(i)]=k_{e}(i)
$$

em que $k_{d}(i)$ é a densidade da corrente de tráfego da faixa da direita (fluxo misto), no intervalo $i$, em veic/(km.faixa). Isolando-se $E(i)$ do lado esquerdo da equação, têm-se que:

$$
E(i)=\frac{1}{p(i)} \cdot\left[\frac{k_{e}(i)}{k_{d}(i)}-1\right]+1
$$

Com a Equação (9), é possível obter uma estimativa do fator de equivalência veicular para cada um dos intervalos de tempo $i, \hat{E}(i)$.

\section{COLETA DE DADOS}

Os dados disponíveis para este estudo caracterizam tanto o tráfego, quanto as condições do tempo (com ou sem chuva) no instante da coleta dos dados. Os dados de fluxo e velocidade foram obtidos por laços indutivos, que permitem obter informações de número de veículos, divididos entre automóveis, veículos comerciais e motos, bem como suas velocidades médias. Essas informações são desagregadas por faixa e os dados são agrupados em intervalos de 5 ou 6 minutos, dependendo da estação de coleta de dados [Andrade, 2012].

Os dados utilizados na pesquisa foram coletados em 8 estações permanentes de monitoramento localizadas em rodovias de pista dupla do estado de São Paulo com três ou mais faixas de tráfego. As rodovias selecionadas para estas pesquisas são a SP280 (Rodovia Castelo Branco) e a SP348 (Rodovia dos Bandeirantes) e os dados foram obtidos nos anos de 2010 e 2011. A Tabela 1 fornece a localização dos pontos e as principais características referentes a cada um deles. A velocidade crítica é aquela que separa o fluxo não congestionado do fluxo congestionado [Andrade e Setti, 2014].

Apenas trechos em aclive foram escolhidos para compor a amostra, uma vez que o efeito dos veículos pesados na qualidade de serviço é mais notado em aclives, pela perda de velocidade que ocorre em subidas. Os dados disponíveis para a realização desta pesquisa não diferenciam caminhões, ônibus e veículos de recreação. Também não é informada a quantidade de eixos de cada veículo, já que a diferenciação entre automóveis e veículos comerciais é feita pelo comprimento dos veículos.

Os dados meteorológicos foram fornecidos pelo Instituto de Pesquisas Meteorológicas da UNESP (IPMet) e 
PIVA, F.J.; SETTI, J.R.

Tabela 1. Localização dos pontos de coleta utilizados na pesquisa

Fonte: [Andrade, 2012]

\begin{tabular}{|c|c|c|c|c|c|c|c|}
\hline Posto & Rodovia & Local & Tipo & $\begin{array}{l}\text { Ocupação } \\
\text { lindeira }\end{array}$ & $\begin{array}{l}\text { Atinge } \\
\text { capacidade }\end{array}$ & $\begin{array}{l}\text { Velocidade } \\
\text { crítica }\end{array}$ & Greide \\
\hline 1 & SP280 & $\begin{array}{l}\mathrm{km} \mathrm{27,0} \\
\text { Leste }\end{array}$ & Autoestrada & Urbana & Sim & $79 \mathrm{~km} / \mathrm{h}$ & $3,5 \%$ \\
\hline 2 & SP280 & $\begin{array}{l}\mathrm{km} 29,5 \\
\text { Leste }\end{array}$ & Autoestrada & Urbana & Sim & $77 \mathrm{~km} / \mathrm{h}$ & $2,0 \%$ \\
\hline 3 & SP280 & $\begin{array}{l}\mathrm{km} 37,0 \\
\text { Leste }\end{array}$ & Pista dupla & Rural & Sim & $90 \mathrm{~km} / \mathrm{h}$ & $5,0 \%$ \\
\hline 4 & SP280 & $\begin{array}{l}\mathrm{km} 51,9 \\
\text { Leste }\end{array}$ & Pista dupla & Rural & Sim & $84 \mathrm{~km} / \mathrm{h}$ & $4,5 \%$ \\
\hline 5 & SP280 & $\begin{array}{l}\mathrm{km} 59,6 \\
\text { Leste }\end{array}$ & Pista dupla & Rural & Não & - & $1,5 \%$ \\
\hline 6 & SP348 & $\begin{array}{l}\mathrm{km} 32,0 \\
\text { Norte }\end{array}$ & Autoestrada & Rural & Sim & $97 \mathrm{~km} / \mathrm{h}$ & $3,5 \%$ \\
\hline 7 & SP348 & $\begin{array}{l}\mathrm{km} 59,0 \\
\text { Norte }\end{array}$ & Autoestrada & Rural & Não & - & $1,0 \%$ \\
\hline 8 & SP348 & $\begin{array}{l}\mathrm{km} 87,0 \\
\text { Norte }\end{array}$ & Autoestrada & Rural & Não & - & $2,5 \%$ \\
\hline
\end{tabular}

consistem em imagens de radares meteorológicos localizados nas cidades de Bauru e Presidente Prudente. As imagens possuem intervalos de capturas de 30 minutos [Pizzol e Setti, 2013]. Esses dados foram correlacionados com os dados de tráfego, de tal forma que foi possível identificar os períodos de ocorrência de chuva nos locais de coleta de dados. Apenas observações de fluxo e velocidade feitas com pista seca foram utilizadas neste estudo.

Conforme apresentado na Figura 2, os caminhões são mais comuns nas faixas mais à direita. Portanto, foram utilizados como fluxo misto $q_{m}$ as observações da faixa mais à direita em todos os pontos de coleta. Além disso, identificou-se que, em alguns trechos estudados, a segunda faixa mais próxima ao acostamento apresentava a mesma qualidade de serviço da faixa da esquerda, em grande parte do tempo. Desta forma, foram incluídas na base de dados observações da segunda faixa mais próxima ao acostamento coletadas pelos sensores instalados na Rodovia dos Bandeirantes (SP348).

\subsection{Tratamento do banco de dados}

Eliminados os intervalos em que houve mau funcionamento dos sensores, bem como as observações realizadas sob condições meteorológicas incertas (isto é, quando não se podia determinar com certeza se chovia ou não), os dados disponíveis foram submetidos a um tratamento que visou remover as observações espúrias - isto é, consideradas inconsistentes, incoerentes ou imprecisas. Para tanto, foram criados critérios apresentados a seguir.

Filtro 1 - Dados coletados sob tempo chuvoso: há uma redução tanto de velocidade de fluxo livre quanto de capacidade com pista molhada [Pizzol e Setti, 2013], as observações de fluxo e velocidade feitas sob chuva ou sob tempo indeterminado (isto é, não se pode garantir que não chovia durante a coleta dos dados) foram excluídas para evitar ruídos nas análises. Com o uso deste filtro, foi identificado que $85,7 \%$ das observações foram coletadas com tempo bom, conforme mostrado na Figura 3.

Filtro 2 - Dados coletados em fluxo congestionado: para não serem utilizadas observações feitas no re- gime de fluxo congestionado (nível de serviço F), foram removidos os dados nos quais a velocidade média na faixa da esquerda é inferior à velocidade crítica que separa o tráfego livre do tráfego congestionado para o trecho em questão [Andrade e Setti, 2014]. A velocidade crítica encontrada variou entre $77 \mathrm{~km} / \mathrm{h}$ e $97 \mathrm{~km} / \mathrm{h}$ e é apresentada na Tabela 1 para cada uma das seções. A aplicação deste filtro mostrou que apenas $2,6 \%$ das observações disponíveis foram coletadas em regime congestionado, conforme indicado na Figura 4.

Filtro 3 - Veículos comerciais na faixa da esquerda: para obter o fluxo básico na faixa da esquerda, foram removidos aqueles intervalos em que foram detectados veículos pesados nesta faixa de tráfego. Assim, pode-se garantir que o fluxo básico é composto apenas por automóveis. A Figura 5 mostra que $26,3 \%$ dos dados continham pelo menos um caminhão na faixa da esquerda durante o intervalo de observação.

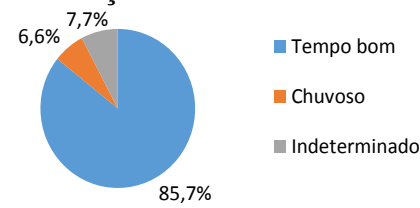

Figura 3. Observações removidas em função das condições meteorológicas

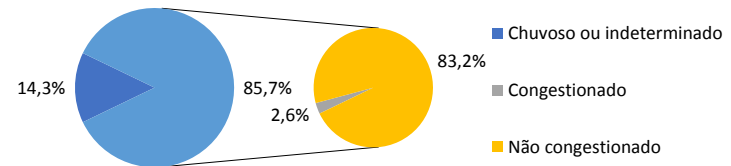

Figura 4. Observações removidas em função da operação em regime de fluxo congestionado (NS F)

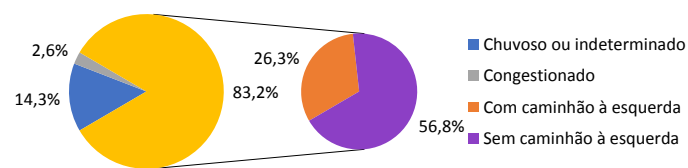

Figura 5. Observações removidas em função da existência de caminhões na faixa da esquerda 


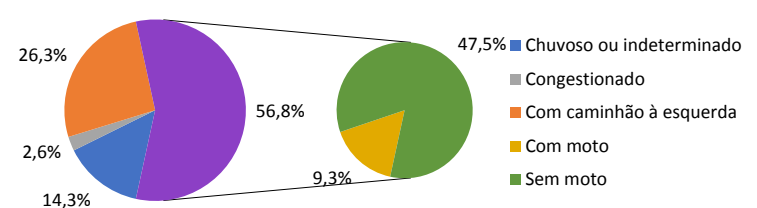

Figura 6. Observações removidas em função da existência de motos na corrente de tráfego

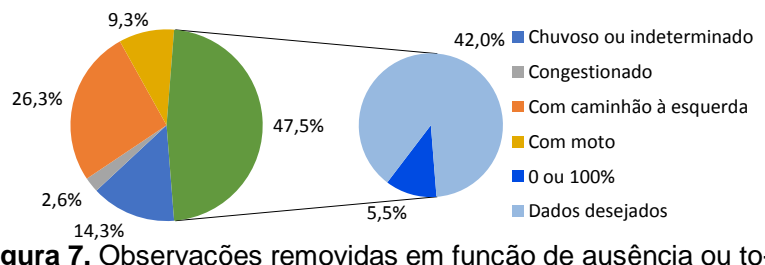

Figura 7. Observações removidas em função de ausência ou totalidade de carros na faixa da direita

Filtro 4 - Motos na corrente de tráfego: em função do comportamento de seus condutores, que muitas vezes preferem viajar entre as faixas de tráfego, é difícil determinar o impacto das motos na corrente de tráfego. Desta forma, foram removidos os intervalos em que era constatada a existência de pelo menos uma moto na corrente de tráfego. Como se mostra na Figura 6, resultou da aplicação deste filtro que $9,3 \%$ das observações foram removidas da amostra por este motivo.

Filtro 5 - Intervalos com $P_{T}=100 \%$ ou $0 \%$ na faixa da direita: para obter-se o fluxo misto na faixa da direita é necessário que os dados contenham tanto carros quanto caminhões. Assim, foram removidos da amostra os intervalos em que a porcentagem de caminhões no tráfego na faixa da direita era $100 \%$ (só de caminhões) ou $0 \%$ (só automóveis). A Figura 7 mostra que 5,5\% dos dados foram removidos nesta etapa do tratamento.

Os filtros 1 a 5 servem para eliminar condições que introduziriam condições não controladas na estimativa do fator de equivalência. Após a aplicação destes filtros restaram 139.253 intervalos de observação, correspondendo a $42 \%$ das observações de fluxo-velocidade disponíveis inicialmente.

Uma análise das cerca de 140 mil observações disponíveis mostrou que, para um número considerável de intervalos de observação (mais da metade dos intervalos restantes), $E(i) \leq 1$. No entanto, valores do fator de equivalência iguais ou menores do que a unidade não fazem sentido, pois caminhões são mais longos que automóveis e têm desempenho inferior. Além disso, nos trechos estudados, a velocidade máxima regulamentada para caminhões é inferior ao limite de velocidade para automóveis, provocando a ocorrência de dois fluxos de tráfego na mesma rodovia: um fluxo de automóveis (mais rápido, nas faixas à esquerda) e um de caminhões, mais lento, nas faixas à direita. Dessa forma, tudo indica que, nos intervalos em que se observa $\hat{E}(i) \leq 1$, a qualidade de serviço no fluxo básico (faixa da esquerda) era diferente da qualidade de serviço no fluxo misto (faixa da direita). Por exemplo, em uma rodovia em que existam $3 \mathrm{veic} /(\mathrm{km}$.faixa) na faixa da esquerda, contendo apenas automóveis e 6 veic/(km.faixa) na faixa da direita, com 50\% de caminhões. Pela Equação (9), tem-se que:

$$
E(i)=\frac{1}{0,5} \cdot\left[\frac{3}{6}-1\right]+1=0
$$

Ou seja, o equivalente veicular para essa situação seria igual a 0 . Neste caso, porém, observa-se que a qualidade de serviço é pior na faixa da direita do que na faixa da esquerda. Em função disso, dois filtros adicionais foram usados para eliminar do conjunto de observações realizadas em condições nas quais a qualidade de serviço não era a mesma na corrente básica (só automóveis) e na corrente mista (automóveis e caminhões).

Filtro 6 - Densidade de tráfego inferior a 3 veic/(km.faixa) em uma das faixas: Feita uma avaliação dos intervalos em que $\hat{E}(i) \leq 1,0$ percebeu-se que a maioria dos dados que eram removidos quando a densidade de veículos era baixa em uma das duas faixas de tráfego (fluxo básico e misto). Esse limiar foi escolhido porque, para este valor, $75 \%$ das observações seriam submetidas ao próximo filtro. Desta forma, decidiu-se remover aqueles intervalos em que $k(i)<3 \mathrm{veic/(km.faixa)} \mathrm{em} \mathrm{uma} \mathrm{das} \mathrm{duas} \mathrm{faixas} \mathrm{de}$ tráfego analisadas, tanto de fluxo misto, quanto de fluxo básico. A Figura 8 apresenta que 20,4\% das observações foram removidas com este filtro. Essa remoção representa quase $50 \%$ dos intervalos que haviam restado após a aplicação do Filtro 5.

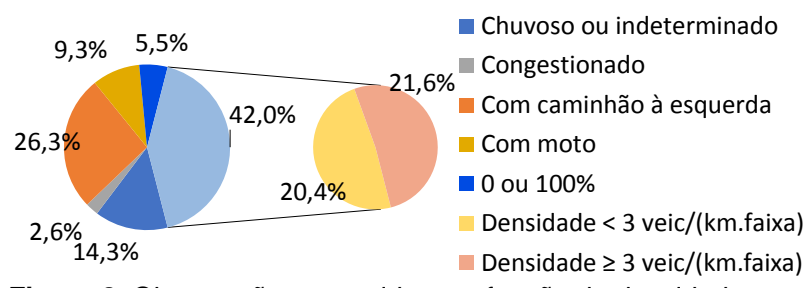

Figura 8. Observações removidas em função de densidade inferior a 3 veic/(km.faixa)

Filtro 7 - Equivalentes veiculares iguais ou menores que 1,0: Após a aplicação do filtro anterior ainda existiam intervalos em que $\hat{E}(i) \leq 1,0$. Assim, os intervalos que ainda apresentavam esses resultados foram excluídos da amostra. A aplicação deste filtro causou a remoção de 5,4\% das observações disponíveis (Figura 9).

Após a aplicação dos sete critérios, têm-se que 16,2\% das observações fluxo-velocidade disponíveis puderam ser efetivamente utilizadas para estimar o valor do fator de equivalência. Essas observações totalizam 53.655 intervalos de curta duração (5 ou 6 minutos). Somados esses intervalos fornecem 312.451 minutos de coleta de dados, ou seja, mais de 200 dias.

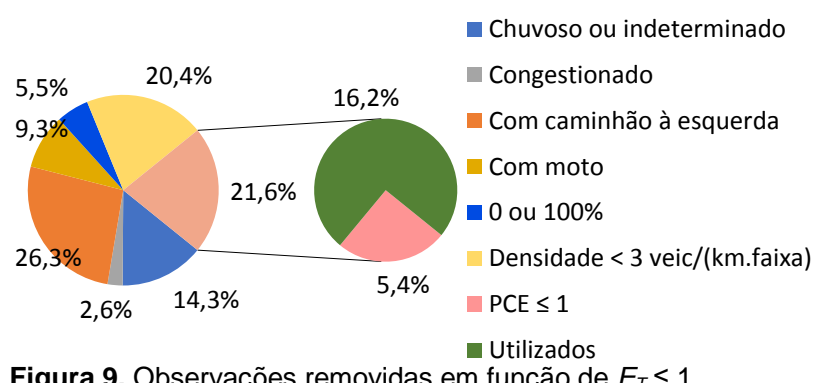

Figura 9. Observações removidas em função de $E_{T} \leq 1$ 


\section{OBTENÇÃO DAS FUNÇÕES DO FATOR DE EQUIVALÊNCIA}

A partir da Equação (9) foram obtidas estimativas do valor do equivalente veicular $\hat{E}(i)$ para cada um dos intervalos de tempo $i$ da amostra. Essas estimativas do fator de equivalência foram agrupadas em função de dois critérios. Primeiramente, os locais de coleta foram agrupados em função da declividade da pista, conforme critério apresentado na Tabela 2 (a). A seguir, os valores estimados para os fatores de equivalência veicular foram agrupados em função do nível de serviço na faixa mais próxima ao canteiro central (densidade $k_{e}$ ), com os limites definidos pelo HCM [TRB, 2010], apresentados na Tabela 2 (b).

Tabela 2. Critérios para classificação dos trechos estudados: (a) em função do greide; e (b) em função do nível de serviço

(a)

\begin{tabular}{cc}
\hline Classe de greide & Declividade (\%) \\
\hline Classe 1 & $0 \%<I \leq 2 \%$ \\
Classe 2 & $2 \%<I \leq 4 \%$ \\
Classe 3 & $4 \%<I$ \\
\hline
\end{tabular}

(b)

\begin{tabular}{cc}
\hline Nível de serviço & Densidade (cpe/(km.faixa) \\
\hline A & $k_{e} \leq 6,8$ \\
B & $6,8<k_{e} \leq 11,2$ \\
C & $11,2<k_{e} \leq 16,2$ \\
D & $16,2<k_{e} \leq 21,7$ \\
E & $21,7<k_{e} \leq 28,0$ \\
\hline
\end{tabular}

$\mathrm{Na}$ abordagem proposta, o valor do fator de equivalência veicular depende de três parâmetros: a fração de caminhões no fluxo da faixa da direita $p(i)$, a densidade da corrente de tráfego na faixa da direita $k_{d}(i)$ e da densidade de tráfego na faixa da esquerda $k_{e}(i)$. Como a composição do tráfego, a velocidade média e o fluxo em cada intervalo $i$ são variáveis aleatórias, então a estimativa $\hat{E}(i)$ do valor do equivalente também é uma variável aleatória. Isso pode ser visto na Figura 10, que mostra os valores estimados do fator de equivalência $\hat{E}(i)$ em função da fração de caminhões $p(i)$, para observações em que a densidade na faixa da direita varia entre 4,5 e 5,5 veic/(km.faixa).

A Equação (9) pode ser usada para estudar a variação do fator de equivalência em função da porcentagem de caminhões, fixando-se os valores de $k_{d}$ e $k_{e}$. A Figura 11 apresenta a variação do fator de equivalência veicular $E(i)$ em função da fração de caminhões na faixa da direita $p(i)$, usando-se a Equação (9) e admitindo-se que a densidade de tráfego na faixa da direita é $k_{d}=5 \mathrm{veic} /(\mathrm{km} \cdot$ faixa), para compatibilização com os dados mostrados na Figura 10. Desta forma, foram obtidas cinco funções diferentes - uma para cada nível de serviço -, variando-se o valor da densidade na faixa da esquerda $k_{e}$. Nota-se que, conforme aumenta a porcentagem de caminhões, mais o valor de $E(i)$ se aproxima de 1 , mostrando que o impacto marginal da adição de veículos pesados na corrente de tráfego é decrescente.

As funções mostradas na Figura 11 são funções hiperbólicas, do tipo

$$
E(i)=a \cdot p(i)^{-b}
$$

em que $p(i)$, a porcentagem de caminhões, é a variável independente e $a$ e $b$ são constantes de calibração. Assim sendo, pode-se ajustar às observações da Figura 10 uma função que representa a variação da estimativa do fator de equivalência em função da porcentagem de caminhões. A Figura 12 mostra a função obtida através de regressão linear para os valores estimados de fatores de equivalentes veiculares para trechos com inclinação entre $2 \%$ e $4 \%$ e nível de serviço C. Para esta função, o coeficiente $\mathrm{R}^{2}$ encontrado é de 0,57 .

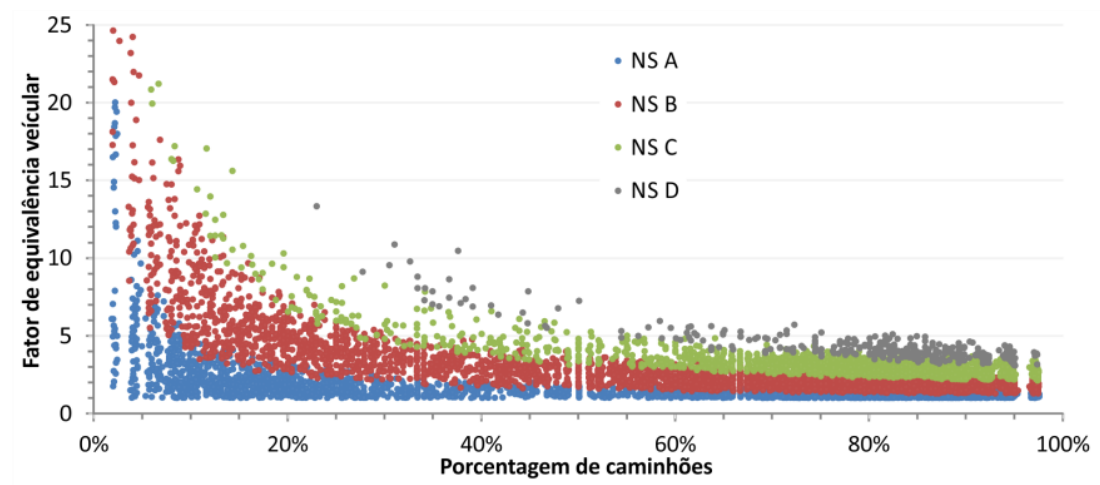

Figura 10. Relação entre as estimativas do fator de equivalência e a fração de caminhões na corrente de tráfego obtidas para densidades na faixa da direita entre 4,5 e 5,5 veic $/ \mathrm{km}$

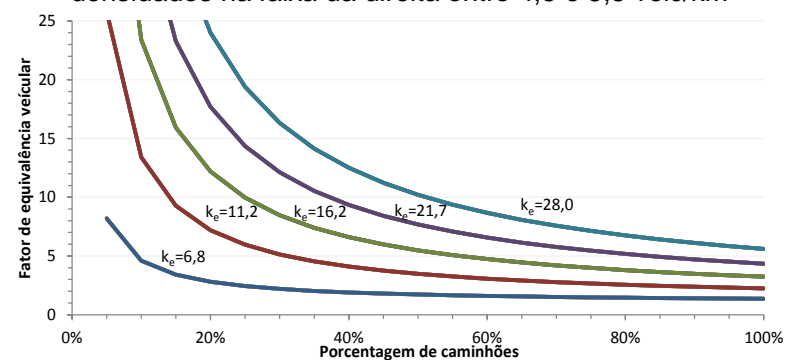

Figura 11. Relação entre fator de equivalência veicular e porcentagem de caminhões na faixa da direita para cinco diferentes densidades na faixa da esquerda 


\section{RESULTADOS EMPÍRICOS}

Para verificar se a abordagem adotada é capaz de identificar corretamente o efeito dos veículos pesados na corrente de tráfego, foi plotada a variação da estimativa do equivalente veicular $\hat{E}(i)$ em função da fração de caminhões para cada uma das classes de greides da amostra.

A Figura 13 mostra as funções com as estimativas dos fatores de equivalência veicular para os locais em que os greides variam entre $0 \%$ e $2 \%$, para níveis de serviços entre A e D. Para porcentagens de caminhões na faixa da direita até $30 \%$ é possível perceber que os níveis C e D apresentam valores do fator de equivalência superiores aos dos níveis A e B, cujas curvas são praticamente coincidentes. Também é possível observar que a partir da fração de $40 \%$ de caminhões os valores de $\hat{E}(i)$ de todos os níveis de serviço se aproximam.

São apresentadas na Figura 14 as funções para trechos com aclives entre $2 \%$ e $4 \%$. Neste caso, pode-se notar que a curva do nível de serviço $B$ apresenta valores de $\hat{E}(i)$ superiores ao nível de serviço A. Esse fenômeno também ocorre com as curvas da Figura 13, embora nos resultados da Figura 14 a diferença seja mais acentuada para porcentagens mais baixas. Pode-se observar também que quanto pior o nível de serviço, maior o fator de equivalência veicular para uma mesma porcentagem de caminhões na faixa da direita.

Na Figura 15 são apresentados os valores de $\hat{E}(i)$ para greides maiores que $4 \%$. Observa-se que para porcen-

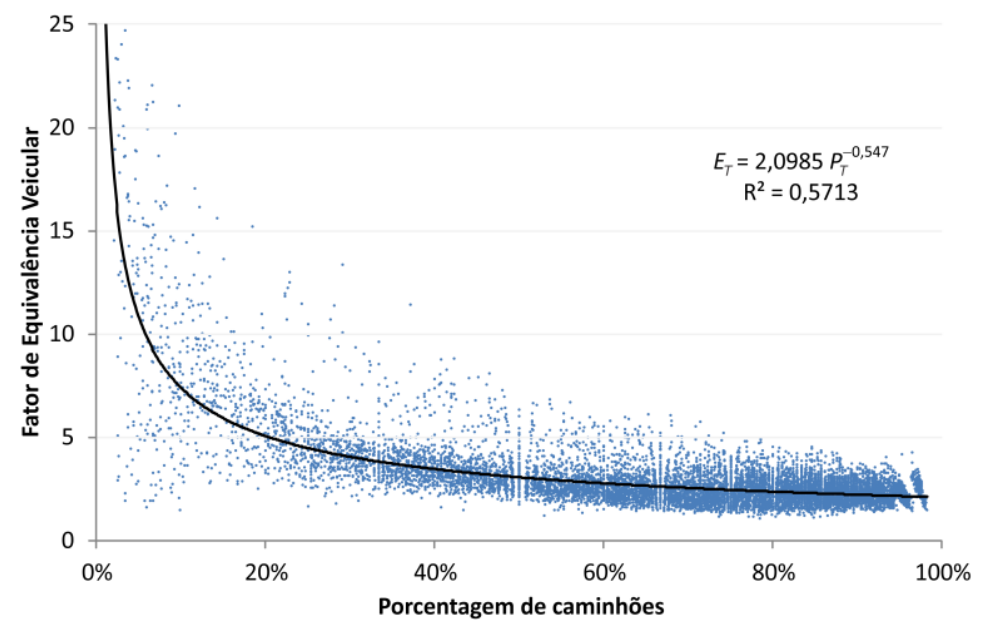

Figura 12. Função ajustada para representar a variação da estimativa do fator de equivalência em função da fração de caminhões na corrente de tráfego, para greides entre $2 \%$ e $4 \%$ e nível de serviço C

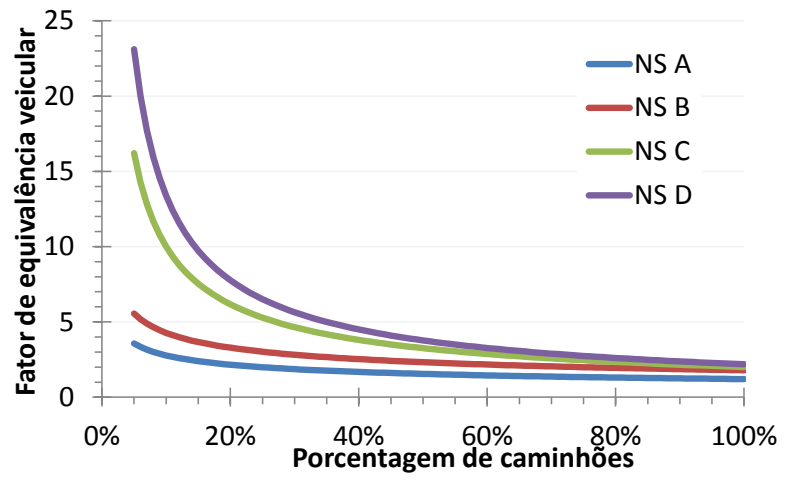

Figura 13. Variação do fator de equivalência em função da porcentagem de caminhões, greides entre $0 \%$ e $2 \%$

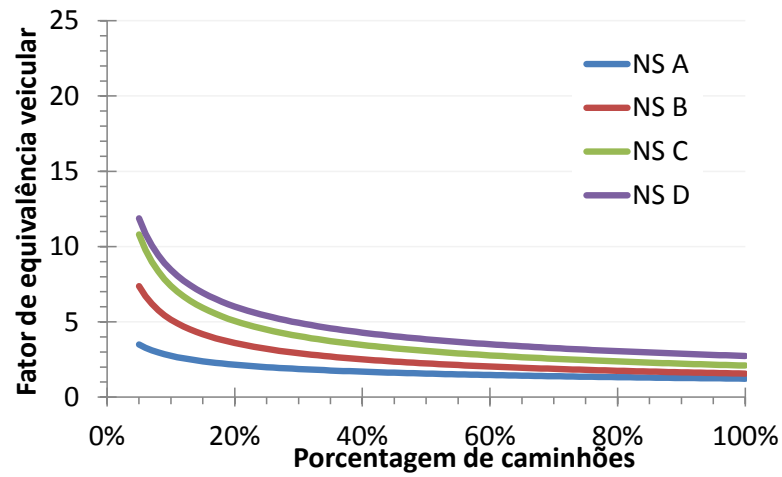

Figura 14. Variação do fator de equivalência em função da fração de caminhões, para greides entre $2 \%$ e $4 \%$ 


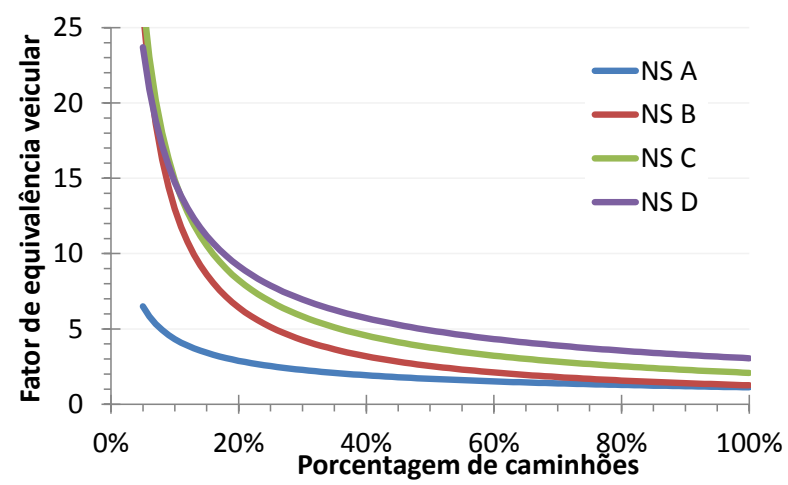

Figura 15. Variação do fator de equivalência em função da porcentagem de caminhões, para greides superiores a $4 \%$

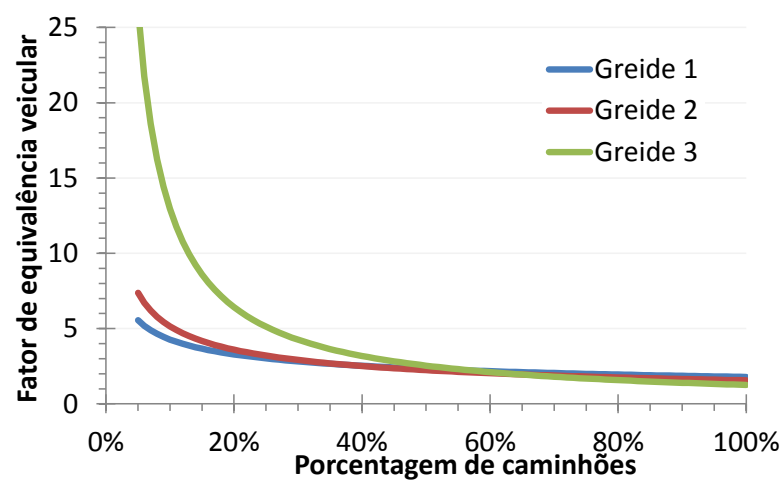

Figura 16. Comparação entre as estimativas de fator de equivalência em função da fração de caminhões e da classe de inclinação nos locais de coleta, para o nível de serviço $B$

tagens de caminhões inferiores a $40 \%$ as curvas para os níveis de serviço B, C e D são praticamente iguais e estão acima da curva para o nível de serviço A.

Também foi feita a comparação entre as estimativas do fator de equivalência veicular para diferentes classes de greides em um mesmo nível de serviço, como apresentado na Figura 16, que mostra as comparações para o nível de serviço B. Pode-se observar que até $50 \%$ de caminhões na faixa da direita, quanto maior a inclinação da rodovia, maior o fator de equivalência veicular para uma mesma porcentagem de caminhões. Para frações superiores a $50 \%$ os valores das estimativas $\hat{E}(i)$ parecem deixar de ser afetados pelo greide.

Para os pontos de coleta utilizados nessa pesquisa, identificou-se que em cinco locais a distância entre o laço indutivo e o início do aclive é menor do que $500 \mathrm{~m}$. Em apenas um caso a distância é superior a 1,25 km. Porém, como foram utilizados apenas dados empíricos de fluxo-velocidade, obtidos através de estações permanentes de monitoramento de tráfego, não foram estudados os efeitos ligados à extensão de rampas.

\section{CONSIDERAÇÕES FINAIS}

Na presente pesquisa, avaliou-se o impacto de veículos pesados na qualidade de serviço através de estimativas do fator de equivalência obtidas a partir de dados de fluxo e velocidade coletados por estações de monitoramento do tráfego em cada faixa de rolamento, a intervalos de tempo curtos (5 ou 6 minutos). Para estimar valores do fator de equivalência, adotou-se o método proposto por Huber [1982], considerando-se o fluxo básico como o da faixa da esquerda, nos intervalos em que são detectados apenas automóveis, e o fluxo misto como o da faixa da direita, em que passam automóveis e caminhões. Dessa forma, é possível estimar o valor do fator de equivalência para um dado intervalo de observação, se for feita a hipótese de que o nível de serviço é o mesmo nas duas faixas de tráfego durante aquele curto intervalo de tempo.

Os resultados da análise dos dados sugerem que a hipótese de que o nível de serviço é constante ao longo das faixas de tráfego da rodovia não é verdadeira em cerca de $52 \%$ das observações disponíveis. Isso pode ser decorrente da existência, nos trechos estudados, de limites de velocidade diferentes para carros de passeio e caminhões, o que cria duas correntes de tráfego de velocidades diferentes que, por vezes, experimentam níveis de serviço distintos. Notase, em todos os casos estudados, que o impacto marginal dos caminhões decresce à medida em que a porcentagem de caminhões na corrente aumenta, conforme a teoria estabelece.

Para trechos planos (greides $<2 \%$ ), notou-se que os valores estimados para o fator de equivalência são praticamente os mesmos para os níveis de serviço A e B; para porcentagens altas de caminhões $(p>40 \%)$, as diferenças devidas ao nível de serviço virtualmente desaparecem. Para os aclives mais íngremes (greides $>4 \%$ ), o efeito do nível de serviço aparece apenas para o nível de serviço A; as curvas para os níveis de serviço B, C e D são muito próximas, para qualquer porcentagem de caminhões. De fato, comparandose as curvas das Figuras 13, 14 e 15, percebe-se que as curvas dos níveis de serviço $\mathrm{B}, \mathrm{C}$ e D vão se aproximando na medida em que os aclives tornam-se mais íngremes, eviden- 
ciando o efeito das limitações de desempenho dos caminhões na qualidade de serviço, quando medido através do fator de equivalência veicular estimado através do método de Huber.

\section{AGRADECIMENTOS}

Os autores agradecem o apoio da CAPES e do CNPq pelo suporte financeiro sob a forma de bolsas de mestrado e de produtividade em pesquisa, respectivamente. Os autores também agradecem à ARTESP e às concessionárias ViaOeste e AutoBan pela disponibilização dos dados de tráfego e ao IPMet, pelo fornecimento das imagens dos seus radares meteorológicos

\section{REFERÊNCIAS}

Andrade, G. R. (2012) Capacidade e relação fluxo-velocidade em autoestradas e rodovias de pista dupla paulistas. Dissertação Mestrado, EESC-USP, São Carlos, SP.

Andrade, G. R. e Setti, J. R. (2014) Speed-flow relationship and capacity for expressways in Brazil. In: Innova-tive Applications of the HCM 2010. Transportation Research Circular E-C190, $T R B$, Washington, DC, p. 10-25.

Cunha, A. L. B. N. e Setti, J. R. (2009) Fatores de Equivalência para Caminhões em Rodovias de Pista Dupla. In: Anais do $6^{\circ}$ Congresso Brasileiro de Rodovias e Concessões, Florianópolis, $\mathrm{SC}$ (CD-ROM), $15 \mathrm{p}$.

Demarchi, S. H. e Setti, J. R. (2003) Limitations of passenger-car equivalent derivation for traffic streams with more than one truck type. Transportation Research Record, v. 1852, p. 96-104.

DOI: $10.3141 / 1852-13$

Demarchi, S. H (2000) Influência dos Veículos Pesados na Capacidade e Nível de Serviço de Rodovias de Pista Dupla. Tese Doutorado, EESC-USP, São Carlos, SP.

Elefteriadou, L.; Torbic, D. e Webster, N. (1997) Development of passenger car equivalents for freeways, two-lane highways, and arterials. Transportation Research Record, v. 1572, p. 51 58 . DOI: 10.3141/1572-07

Huber, M. J. (1982) Estimation of passenger-car equivalents of trucks in traffic stream. Transportation Research Record, v. 869, p. 60-70.

Linzer, E. M., Roess, R. P. e McShane,W. R. (1979). Effect of trucks, buses, and recreational vehicles on freeway capacity and service volume. Transportation Research Record, v. 699, p. 1725 .

Pizzol, B. e Setti, J. R. (2013) Fusão de bancos de dados para estudo do efeito das condições meteorológicas no fluxo de veículos em rodovias paulistas. In: Anais do XXVII Congresso de Pesquisa e Ensino em Transportes (CD-ROM), ANPET, Belém, PA.

Riente, G.; Rodrigues-Silva, K. e Puty Filho, S. (2011) Panorama Normativo e Tecnológico da Avaliação Operacional das Concessões Rodoviárias. In: Anais do $7^{\circ}$ Congresso Brasileiro de Rodovias e Concessões, Foz do Iguaçu, PR (CD-ROM), 14 p.

Setti, J. R. (2009) Highway Capacity Manual ou Manual de Capacidade Viária? In: Anais do $6^{\circ}$ Congresso Brasileiro de Rodovias e Concessões, Florianópolis, SC (CD-ROM), 11 p.

TRANSPORTES v. 23, n. 4 (2015), p. 51-59
Sumner, R.; Hill, D. e Shapiro, S. (1984) Segment passenger car equivalent values for cost allocation on urban arterial roads. Transportation Research A, v. 18-A, n. 5/6, p. 399-406. DOI: 10.1016/0191-2607(84)90014-1

TRB (2010) Highway Capacity Manual 2010. Transportation Research Board, National Research Council, Washington, DC, EUA. 